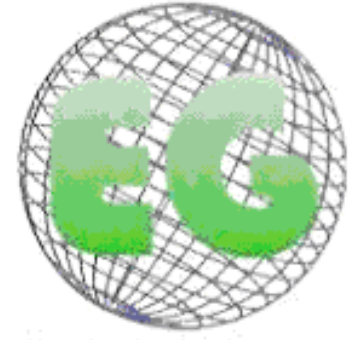

ISSN 1695-6141

\title{
Surgimiento y desarrollo de la Historia de la Enfermería en España
} Rise and development of the History of Nursing in Spain

\section{*García García, Inmaculada **Gozalbes Cravioto, Enrique}

\author{
*Doctora por la Universidad de Granada. Profesora Departamento de Enfermería. Universidad de \\ Granada. E-mail: igarcia@ugr.es ** Doctor por la Universidad de Granada. Profesor Departamento de \\ Historia. Universidad de Castilla la Mancha.
}

Palabras clave: Historia de la Enfermería; Historia de la Medicina; Historia de la Salud; España. Keywords: History of Nursing; History of Medicine; History of Health; Spain

\section{RESUMEN}

En el presente trabajo se abordan diversas cuestiones referidas a la investigación acerca de la Historia de la Salud en España. En especial se analizan los avances producidos en España a partir de dos factores principales: los influjos culturales de su entorno europeo, así como las demandas sociales que han conducido a la propia formación universitaria en Medicina y en Enfermería. En el trabajo se aborda la existencia de una cierta tradición profesional y de estudios, hasta ahora poco conocida, pero es tenida en cuenta de forma creciente, así como la incorporación del moderno modelo de interpretación de la Historia Social de la Enfermedad. En este contexto se produce en España el desarrollo desde la Universidad de la investigación en Historia de la Enfermería, y se plantean algunos avances de conocimiento en diversos temas concretos, producidos en los últimos años.

\begin{abstract}
In this paper we study different issues related to the research about the History of Health in Spain. In particular, we analyse the produced achievement in Spain from two main factors: the cultural influences of European environment and social demands that have led to the university education in Medicine and Nursing. The paper examines the existence of a professional tradition and studies, hitherto little known, but that it is being increasingly taken into account. At the same time, the paper studies the incorporation of modern interpretation model of the Social History of the Disease. In this context the University development of research in the History of Nursing is produced in Spain and some progress of knowledge in several specific items produced in recent years are considered.
\end{abstract}

\section{INTRODUCCIÓN}

La Historia de la Salud en España en los siglos XIX y XX ha evolucionado, como no podía ser de otra forma, a partir de dos elementos fundamentales: 
a) Por un lado, los influjos culturales de su entorno europeo al que ha estado más vinculada, los avances del conocimiento producidos en esta zona, así como de la práctica de la ciencia y de la investigación en esos países próximos (principalmente Francia, Reino Unido o Alemania).

b) Por el otro, las demandas sociales en el avance hacia la modernidad y la extensión de la Salud Pública, canalizadas a través de las actuaciones políticas de los diferentes gobiernos o administraciones a partir de las transformaciones del Estado liberal (desde 1833) ${ }^{(1)}$.

La conjunción de ambos factores se ha canalizado en la formación de los profesionales y en la mayor parte de las investigaciones desarrolladas en las Universidades. El estudio de la evolución histórica nunca ha sido una prioridad en el terreno de la Salud y de la Ciencia, en consecuencia, la incorporación de la materia a las Facultades de Medicina constituyó un hecho tardío, aún más, la Historia de la Enfermería en su correspondiente título universitario. La primera Cátedra de Historia de la Medicina se dotó en la Universidad Complutense (entonces llamada Central) en el año 1921, y la ocupó el Dr. Eduardo García del Real, y la extensión de cátedras a otras universidades españolas se produjo mucho más tarde ${ }^{(2)}$.

El objetivo de este estudio es analizar el contexto histórico en el que surgió y desarrolló la Historia de la Enfermería

\section{REPLANTEAMIENTO DE UNA OBSERVACIÓN GENERAL}

Pese a lo indicado, en la tradición española existían tratados relevantes no sólo de Medicina, sino también de Historia de esta materia. Ese desarrollo tardío ha sido todavía mayor respecto a otros profesionales de la Salud, en especial de la Enfermería, cuya incorporación como contenido puramente universitario ha sido muy reciente en España, donde hasta hoy se ha considerado un conocimiento "técnico" (3). La Historia de la Enfermería, desarrollada en los últimos años, no deja de ser un simple sub-producto de la tradicional Historia de la Medicina, lo cual se observa en los recientes Congresos Españoles de Historia de la Enfermería (el primero celebrado en Valencia en octubre de 1994), que siguen el modelo de los más clásicos Congresos de Historia de la Medicina (primer Congreso celebrado en Madrid-Toledo en abril de 1963).

Muchas veces se ha insistido en el atraso español en materia de Ciencia, producto de elementos como la Inquisición y la crisis del siglo XVII. Dicha concepción, con sus argumentos a favor, forma parte de la visión general pesimista de la Historia de España. Pero en el terreno más objetivo de la propia Historia, el atraso de las Ciencias en España, del que han sido plenamente conscientes los médicos escritores $^{(4)}$, no sería otra cosa que el producto del fracaso general en muchos aspectos (de la revolución liberal, de la revolución industrial, de la democracia, de la modernización, etc.). Fracaso que tendría su resultado final en el hecho de que tan sólo dos españoles, Santiago Ramón y Cajal y Severo Ochoa, hayan accedido al Premio Nobel en Ciencias.

No obstante, en las últimas décadas, a partir del éxito del cambio político y social de la Transición, así como de la incorporación a la construcción europea, los hechos han comenzado a observarse de forma diferente. Este replanteamiento de conjunto de la Historia de España, muy animado por los hispanistas franceses y anglosajones, que 
sobre todo en los últimos tiempos llamaban nuestra atención acerca de que sus respectivas Historias no habían sido tampoco remansos de paz y de avances continuos. Se ha abierto paso la necesidad de la observación más objetiva de la Historia, sin tanta autocrítica, revisión histórica que se extiende a diversos pasajes leídos de forma tópica ${ }^{(5)}$.

\section{EXISTENCIA DE UNA TRADICIÓN PROFESIONAL Y DE ESTUDIOS}

La nueva mirada a la Historia de la Ciencia en España, y de la Historia de la Salud y de la Medicina, ha permitido comenzar a revisar algunas cuestiones. Como muy bien ha detectado Barona ${ }^{(6)}$, la evolución del análisis histórico de la ciencia, así como de la salud y del tratamiento de la enfermedad y de los enfermos en España, se ha integrado desde el siglo XVIII (cuando la llustración tuvo fuerte presencia en el país) en la tradición europea del estudio histórico de la ciencia, en general, y de la medicina y de la salud, en particular. Muchas veces sin terminar de consolidar la línea emprendida, pero desde el poder político se emprendieron campañas relativamente exitosas, si bien de una continuidad limitada, y el influjo de médicos y científicos de otros países europeos en algunos homólogos españoles también parece indudable en muchos momentos.

Esta constatación menos pesimista, intentando no caer en el peligro contrario del péndulo, está permitiendo la recuperación de la memoria perdida. Existe un campo en el que esta recuperación es particularmente evidente: la del exilio español en México, en Francia o en la República Argentina, a consecuencia de la guerra civil (1936-1939). Una parte de la intelectualidad y de la ciencia española se había decantado por la República democrática, y el triunfo franquista en la guerra supuso la persecución, la depuración y en muchos casos, la necesidad del exilio ${ }^{(7)}$.

Según el censo establecido por $\operatorname{Ordóñez}^{(8)}$, nada menos que 325 científicos españoles se establecieron en el exilio mexicano, destacando entre ellos la fortísima proporción de los médicos (el 43\%). Y ello fue también paralelo a otro de los aspectos incorporados a la investigación en esta misma línea de recuperación de la memoria: la depuración de las Universidades por parte del régimen triunfante. $Y$ entre ellas, el significativo caso de la Facultad de Medicina de Madrid, cuyos profesores sufrieron de forma muy intensa el desarrollo y los resultados de la conflagración ${ }^{(9)}$.

Este ejemplo de recuperación de la memoria sepultada es similar al que también la investigación histórica realiza actualmente hacia tiempos mucho más pretéritos. El citado Barona destaca justamente el caso de la llustración, que ha atraído la atención de numerosos investigadores en España, y ha destacado escritos como los del médico Piquer, o el caso de Quer, defensor de la realidad de la ciencia española en la época ${ }^{(6)}$. Y también las actuaciones y prácticas de numerosos médicos que han sido estudiados desde los voluminosos y bien documentados trabajos al respecto de Granjel ${ }^{(10)}$.

Aún así, muchas veces esos avances científicos se produjeron en el ámbito individual y puramente voluntarista, por tanto con unos alcances muy limitados, y sobre todo, en esa época se produjo lo que se ha llamado la "militarización" de la ciencia española, muy ligada por ejemplo a las iniciativas de los marinos ${ }^{(11)}$. Resulta indudable que el nuevo desarrollo que se detecta en la medicina española del siglo XVIII alcanzó de forma muy directa en sus efectos al Nuevo Mundo, y así ha sido bien destacado por 
ejemplo en relación con Chile ${ }^{(12)}$, y también en lo que respecta a la práctica y desarrollo de la medicina en Mexico ${ }^{(13)}$.

El análisis de la antigua literatura permite observar la existencia de una larga tradición que en el terreno médico, más allá de la aportación más conocida y valorada de Miguel de Servet, incluye otras obras olvidadas pero muy importantes como avanzadilla de la ciencia en su época, como el tratado de actuación médica de Chirino ${ }^{(14)}$, la medicina que se practicaba en Sevilla a mediados del siglo XVI descrita por Aviñón (15), el importante tratado de Laguna dedicado a las medidas para preservar y luchar contra las epidemias de peste bubónica ${ }^{(16)}$, o los diversos tratados de Lobera de Ávila, entre ellos el dedicado a la atención de las distintas enfermedades ${ }^{(17)}$.

Y por supuesto también, esta nueva mirada se dirige en relación con la Historia de la Enfermería, y de los cuidados de enfermería. Una acción de enfermería en realidad muy especializada, ligada sobre todo a la actuación de los religiosos en los hospitales. Con todo, cabe indicar que esta actuación en realidad alcanzó una fuerte especialización sanitaria. Esa fuerte especialización profesional de los enfermeros en buena parte en España se perdió en la crisis del siglo XIX, aunque es cierto que esta tesis pueda ser discutida. Pero hay datos de potente especialización en los siglos XVI al XVIII, en la actuación en los hospitales existentes en España.

Existen notables casos de tradición en el trabajo proto-profesional y especializado con los enfermos. En especial, la Congregación de los Hermanos de Bernardino de Obregón, cuya Instrucción para enfermeros y consuelo de los afligidos enfermos de 1617 tuvo también su importante traslación a la atención sanitaria en el Nuevo Mundo(18). Y también la actuación sanitaria de San Juan de Dios en el siglo XVI en Granada, el patrono de la Enfermería española. La labor heroica y sacrificada o el consuelo a los "afligidos enfermos", más allá de la faceta de los cuidados, se relaciona directamente con ese modelo "religioso" que basaba su actuación sobre todo en el consuelo espiritual respecto al dolor y la salvación del alma.

Es cierto que todo no será sacrificio religioso, sino que también la actuación de enfermería buscará cierta estructuración y sistematización en los cuidados. Este hecho también se detecta en algunos documentos españoles, como por ejemplo en el Directorio de López, dirigido a un "hospital incógnito", pero en el que actuaban los jesuitas. Como señala el propio título de su instrucción, se trataba de un Directorio de enfermeros y artífice de obras de caridad para curar las enfermedades del cuerpo con la práctica de hacer aplicar las medicinas que ordenan los médicos, con el "mejor arte y méthodo que ai en ella ${ }^{(19)}$. Se trata de un escrito de mediados del siglo XVII, hecho por su autor a partir de una larga experiencia de trabajo en los hospitales de Valladolid y Salamanca. La aportación estaba dedicada a todos los que con caridad actuaban en el sacrificio que suponía el cuidado de los enfermos.

Otra parte de la mirada en la recuperación de la tradición de los estudios puede dirigirse también en relación con la propia Historia de la Salud, hasta hace bien poco eclipsada por la Historia de la Medicina. Precisamente los orígenes de la Historia de la Salud en España hay que buscarlos en una obra concreta al respecto de esa temática más preocupante, la que gira en torno a las pestes y epidemias, que en 1803 publicará Villalba Guitarte ${ }^{(20)}$.

Así pues, la primera Historia más o menos completa en relación con las ciencias sanitarias en España se centró en la epidemiología, y más en concreto en la 
inquietante peste que cada cierto tiempo asolaba el imperio español y otros países del entorno mediterráneo. Villalba Guitarte indicaba que la reciente pandemia de su época había venido de América: ¿Qué extraño pues será que en los buques de aquellos países sus tripulaciones, sus ropas, sus cargamentos, se nos haya introducido semejante plaga?... .....No es inverosímil que esta vez haya venido de la América, pues muy pocos días antes de declararse la epidemia en Cádiz, habían entrado dos buques mercantes de aquella región. El autor recurría al nacionalismo para analizar el pasado desde una perspectiva patria: del saludable suelo de España rarísima vez brotarían las funestas ramas de la peste, si por nuestro descuido una semilla adventicia no lo alterase y corrompiese de tiempo en tiempo.

En el desarrollo clásico de la Historia de la Medicina influirán dos tratados de carácter clásico en los que se intentaba sintetizar los escritos que se habían realizado en tiempos anteriores. En este sentido serán representativos dos trabajos que podemos considerar monumentales debido a la cantidad de información recogida, así como a la extensión de la exposición realizada. Nos referimos, en primer lugar, a la historia bibliográfica en 7 volúmenes de Hernández Morejón ${ }^{(21)}$, y a los 8 volúmenes de los Anales históricos publicados por Chinchilla, y de los que cuatro estaban dedicados de forma específica a la historia de la medicina española ${ }^{(22)}$.

El análisis de los fundamentos de la obra de Hernández Morejón muestra la propia funcionalidad de su aportación: un cuadro que representa en un punto de vista la sabiduría, errores, conducta moral y política de los médicos de todas las edades y pueblos, que ofreciendo a los presentes ejemplos que imitar, y desengaños de no aprovecharse, viene a ser escuela de filosofía práctica que enseña a mejorar y aún perfeccionar a los médicos, no sólo en la parte literaria, sino que también en la moral y política. Como puede observarse, el planteamiento que se realiza es desde el interior de la profesión médica, y el protagonismo del estudio se ha centrado en las actuaciones más o menos virtuosas de los médicos. Esta perspectiva es la que va a marcar de forma decidida el planteamiento de la Historia de la Salud en España.

\section{LA CONCEPCIÓN SOCIAL DE LA HISTORIA DE LA SALUD}

Los cambios producidos en la investigación no arrancan de una causa única sino de una pluralidad de elementos. Sin duda, el principal de ellos deriva de la propia transformación de la disciplina de la Historia a lo largo del siglo XX. La vieja historia política o narrativa, basada en los hechos individuales o en las naciones como entidad orgánica, había dejado paso a un tipo de Historia diferente. Desde mediados del siglo XX la Historia había transformado sus miradas para enfocar la mayor parte de las materias objeto de análisis desde la perspectiva social, tanto entendida en el terreno de las clases sociales (grupos verticales), como de los grupos horizontales (sectores sociales, grupos de edad...) o las miradas sobre la vida cotidiana ${ }^{(23,24)}$.

No tenía mucho sentido que la Historia se hubiera transformado y esa nueva perspectiva no llegara al terreno de la Historia de la Ciencia y de la propia Medicina. En efecto, la Historia basada en el protagonismo de los profesionales médicos presentaba en sí misma un arcaísmo bastante similar al que se hallaba en la Historia fundamentada en los grandes personajes. La transformación del objeto de la Historia no podía menos que, aunque de forma paulatina, llegar al terreno del análisis histórico de las cuestiones sanitarias ${ }^{(25)}$. 
Los planteamientos comenzarán a extenderse por Estados Unidos y otros países europeos a partir de mediados de los años sesenta, a partir de las sugerencias de Sigerist ${ }^{(26)}$, y con los estudios de Ackercknecht ${ }^{(27)}$, que planteará la necesidad de centrar la mirada en la afección social de las enfermedades ${ }^{(28)}$. En esta perspectiva tendrá una notable importancia el impacto de la epidemiología; en efecto, las pandemias favorecían que la enfermedad, y la lucha frente a la misma, pudieran ser analizadas no desde la perspectiva individual sino desde la colectiva. Los ejemplos al respecto de la continuidad de estos trabajos, en relación a las grandes epidemias en la Historia, o en el impacto de las enfermedades en las sociedades, y de la lucha contra las mismas, son muy evidentes y no creemos necesario insistir más en la cuestión.

Nos interesa al respecto el impacto de estas nuevas perspectivas en los estudios realizados en España. El propio y máximo representante de la corriente tradicional "técnica" en el análisis de la Historia de la Medicina, nos referimos a Pedro Laín Entralgo, hacia finales de los años sesenta lo planteaba en palabras recogidas tiempo más tarde por el citado López Piñero ${ }^{(2)}$ : durante mucho tiempo la Historia de la Medicina ha sido estudiada sobre todo desde un enfoque bibliográfico o desde el punto de vista de las instituciones médicas. En la actualidad hay un interés creciente en la historia de las ideas médicas... se ha descuidado la historia del enfermo, es decir, de la sociedad que el enfermo representa, así como las numerosas relaciones entre la sociedad y el médico.

Lopez Piñero ha sido probablemente respecto a la investigación española el máximo representante del tránsito, en el último cuarto del siglo $X X$, de la Historia de la Medicina a la Historia Social de la Salud o de la Enfermedad. La nueva atención en España estará provocada sobre todo no sólo por la transformación de la historiografía en general, o por el influjo europeo y norteamericano sino, sobre todo, también debido a las nuevas preocupaciones por las enfermedades. La expansión del SIDA mostrará que las grandes pandemias, que se consideraban definitivamente superadas, ni mucho menos lo estaban tanto ${ }^{(29)}$. Así pues, esa perspectiva se ha renovado de forma amplia, de manera que el protagonismo de los médicos ha dejado paso a la Historia de la Salud ${ }^{(30)}$, y también en lo referido a la investigación de la misma ${ }^{(31)}$.

\section{LA HISTORIA DE LA ENFERMERÍA}

La Historia de la Enfermería constituye un elemento nuevo en España debido a que la formación en esta disciplina tiene un reconocimiento universitario muy reciente en España ${ }^{(32,33)}$. No está de más recordar que en la España del siglo XVIII se expande la necesidad de tratamiento sanitario, pero el mismo tiene un fortísimo déficit de elementos con una formación precisa en la Universidad. La necesidad creará a los trabajadores para atender las mismas, médicos subordinados sin esos estudios en la Universidad, que serán muy numerosos en España y en los distintos países de América Latina. Se trata de los sangradores e incluso de los llamados popularmente ministrantes, ambos médicos sin titulación ${ }^{(34)}$ formados en la práctica con médicos profesionales, y que actuarán como auxiliares, como sangradores o como sanadores populares (romancistas), que en la legislación española tendrán su unificación en el año 1845. En el año 1857 la Ley de Bases para la Instrucción Pública, conocida como Ley Moyano por el nombre del ministro que la auspició, estableció la creación de los denominados Practicantes, que agrupaban a los anteriores, más Callistas, Dentistas (hasta 1877) y Asistentes a partos. 
En esta evolución, "practicantes" y "matronas" formarán sus respectivas asociaciones, y actuarán en los hospitales y en la incipiente sanidad pública. De acuerdo con las normativas establecidas en Francia y otros países algunos años atrás, en 1915 se creó en España el Título de Enfermería; los enfermeros/as agrupaban a los Practicantes (hombres), las Enfermeras (mujeres) y a las Matronas. En 1953, dado que en la práctica no se había desarrollado la normativa, se produce la unificación de estos tres profesionales con el nacimiento de los ATS (Ayudantes Técnicos Sanitarios). Finalmente, a partir de la primera mitad de los años ochenta la integración definitiva en la Universidad convertirá a los antiguos ATS en Diplomados Universitarios en Enfermería ${ }^{(32)}$, que en fechas muy recientes, con la aplicación en España de la Reforma del Espacio Europeo de la Educación Superior, ha conducido al Grado en Enfermería.

En los últimos años el desarrollo de la Historia de la Enfermería muestra su presencia y su propia potencialidad en relación con diversos temas. El propio hecho de un desarrollo reciente de la propia historiografía de la Enfermería, muy ligada también a su enseñanza universitaria, influye en que la perspectiva central del enfermo y la enfermedad esté presente en muchos de los análisis. En este sentido pueden mencionarse los estudios de conjunto sobre la Historia de la Enfermería, que parten desde concepciones o actuaciones primitivas o pre-profesionales, hasta enlazar con los modernos modelos de cuidados. En esta línea destacamos aportaciones relevantes como las de Eseverri ${ }^{(35)}$, Santo-Tomás ${ }^{(36)}$, Siles ${ }^{(37)}$, Hernández Conesa ${ }^{(38)}$, Hernández Martín ${ }^{(39)}$, o García y Martínez ${ }^{(40)}$.

Otro elemento que muestra el desarrollo de la visión histórica de la Enfermería española es el de la celebración reglada en el tiempo de las distintas ediciones de los Congresos Nacionales de Historia de la Enfermería, similares a los de Medicina. Los mismos están contribuyendo a la elaboración de una Historia de la Enfermería en sus distintas temáticas. Un ejemplo significativo lo encontramos en la publicación de las distintas contribuciones del Congreso de Historia de la Enfermería que se celebró en el año 2008 en Almería ${ }^{(41)}$.

Complementario con los elementos anteriores debemos destacar el surgimiento y la consolidación de algunas publicaciones periódicas o revistas especializadas en Enfermería, y en cuyas páginas tienen acogida los análisis históricos sobre avances en los conocimientos, o sobre la propia evolución profesional y del pensamiento. Nos referimos especialmente a publicaciones como Index de Enfermería o Hiades, con especial atención en el primer caso al rigor científico y a la preocupación por el factor "impacto de revistas" (42), mientras la segunda incorpora en la Red un elenco bibliográfico de gran utilidad en relación con de Historia de la Enfermería ${ }^{(43)}$.

Todos estos elementos influyen, sin duda, en que en la actualidad la Historia de la Enfermería en España disponga ya de una producción bibliográfica bastante relevante $^{(44)}$. La revisión bibliográfica permite señalar algunas de las líneas principales de investigación que se desarrollan en los últimos años:

-. Desarrollo de los estudios regionales o locales, que en ocasiones arrancan de estudios de casos realizados a partir de los archivos locales de los siglos XVII y XVIII. Entre estos trabajos podemos destacar análisis sobre Comunidades Autónomas, como en el caso de Castilla-La Mancha ${ }^{(45)}$, o en el caso de provincias el estudio realizado sobre Badajoz ${ }^{(46)}$. 
-. Análisis de literatura "interna" del colectivo de los enfermeros, en especial de las revistas profesionales de Enfermería, en su mayor parte publicadas como órganos de expresión en algunas provincias españolas en los siglos XIX y XX. A través de estas revistas y boletines se filtran numerosos problemas sanitarios del momento, junto naturalmente a la situación de los profesionales. Entre estas aportaciones pueden destacarse los catálogos de Álvarez Nebreda ${ }^{(47,48)}$.

-. Línea de investigación importante es la relacionada con la feminización de la profesión de Enfermería. Entre las numerosas aportaciones a este respecto puede mencionarse el ya citado volumen en que se recogen las aportaciones en el Congreso de Historia de la Enfermería Española de Almería, con aportaciones como la de Lozano Peña acerca de las mujeres en los establecimientos benéficos de Granada en la Edad Moderna, la de Segura Graíño sobre las mujeres y la sanación, la de Claret y García Martínez sobre la contribución de las mujeres cuidadoras en la asistencia sanitaria, o la de García Pardo sobre la mujer y la asistencia en los siglos XV y XVI en Almería ${ }^{(41)}$.

-. Los hospitales considerados como unidad de actuación sanitaria, por un lado, con trabajos como los de López Terrada ${ }^{(49)}$ y de Gallent Marco ${ }^{(50)}$. Por otra parte no sólo se analizan las cuestiones de atención, cuidados, dietética, sino también otros aspectos referidos a la estructura formal o incluso a cuestiones artísticas, todo ello integrado en el concepto de arquitectura asistencial, de lo que es buen ejemplo la monografía de Cambil Hernández ${ }^{(51)}$.

-. La imagen de la profesión presente en el Arte y en las representaciones gráficas, como es el caso del estudio a partir de los sellos de correos de la representación de la Enfermería española ${ }^{(52)}$.

\section{CONCLUSIONES}

El análisis realizado creemos que permite trazar una visión general acerca de la evolución de la Historia de la Salud en España. Tal y como señalamos existe una notable tradición al respecto que arranca del siglo XVI, pero que en la época de la Ilustración, si bien en actuaciones científicas y médicas parciales, recuperaría una parte del terreno perdido en la centuria anterior. Por otra parte la investigación en la Historia de la Salud arrancará desde los comienzos del siglo XIX, y desde su inicio adquirirá la misma característica dominante hasta hace tres décadas: la Historia de la Salud será la Historia de la Medicina, y más en concreto centrará su atención en el protagonismo del profesional médico.

Los cambios sociales, de un lado, y en la historiografía del otro, auspiciarán que este panorama de la "época técnica" se llegue a mostrar anticuada. Junto a ello, el propio peso de las transformaciones experimentadas en la investigación en otros países europeos y en Estados Unidos. De la Historia personal se transitará a la Historia social, en el sentido de la sociedad como la verdadera protagonista de la atención a partir de la incidencia del mal de la enfermedad. De esta forma en España, sobre todo a partir de los años ochenta del siglo XX, comenzará a desarrollarse una concepción social de la Historia en los temas sanitarios.

Finalmente, en el análisis recogemos el papel de las profesiones sanitarias que en absoluto son nuevas, pero sí son profesionalmente emergentes. Esta presencia ya no sólo auxiliar, sino estrictamente profesional, con evidente formación universitaria, ha conducido a que como en otros países, la Enfermería (como ejemplo más 
significativo) haya comenzado a ser objeto de investigación. Una atención de los cuidados y presencia de la Enfermería que se ha centrado en su presencia a lo largo de la Historia, en casos locales o regionales, pero también en aspectos de su surgimiento, desarrollo o representación profesional, a lo largo de los siglos XIX y XX.

\section{REFERENCIAS}

1. Barona JL, Bernabéu-Mestre J. La salud y el Estado. El movimiento sanitario internacional y la administración española (1851-1945). Valencia: Universidad; 2008.

2. López Piñero JM. Pedro Laín Entralgo y la historiografía médica. Madrid: Real Academia de la Historia; 2005: 45.

3. Martínez Martín ML, Chamorro Rebollo E. Historia de la Enfermería. Evolución histórica del cuidado enfermero. Barcelona: Elsevier; 2011.

4. Sosa Velasco AJ. Médicos escritores en España, 1855-1955. Nueva York: Tamesiswoodbrige; 2010.

5. Iglesias C. No siempre lo peor es cierto. Estudios sobre Historia de España. Madrid: Galaxia Gutemberg; 2009.

6. Baraona JL. Ciencia e Historia. Debates y tendencias en la historiografía de la ciencia. Valencia: Universidad; 1994.

7. Otero Carvajal LE. (Dir.). La destrucción de la ciencia en España. Depuración universitaria en el franquismo. Madrid: Editorial Complutense; 2006.

8. Ordóñez Alonso M. Los científicos del exilio español en Mexico: un perfil. Proyecto Clío. Portal Memoria del exilio. Disponible en http://clio.rediris.es/articulos/cientificos.htm. Consultado el 10-V-2012.

9. Pérez Peña F. Exilio y depuración política en la Facultad de Medicina de San Carlos. Madrid: Visión Net; 2005.

10. Granjel LS. La medicina española del siglo XVIII. Salamanca: Universidad; 1979.

11. Martínez Ruiz E, Pazzis Pi E. (Eds.) Ilustración, ciencia y técnica en el siglo XVIII español. Valencia: Universidad; 2008.

12. Cruz Coke R. Historia de la Medicina chilena. Santiago de Chile: Andrés Bello; 1995.

13. Viveros Maldonado G. Hipocratismo en Mexico. Siglos XVI al XVIII. Mexico: Universidad Nacional Autónoma; 2007.

14. Chirino A. Tratado llamado menor daño de la Medicina. Sevilla: 1519.

15. Aviñón J. Sevillana medicina. Sevilla: 1545.

16. Laguna A. Discurso breve sobre la cura y preservación de la pestilencia. Amberes: 1556.

17. Lobera de Ávila L. Libro de las quatro enfermedades cortesanas que son catarro, gota arthetica, sciatica, mal de piedra y de riñones e hijada e mar de buas, y otras cosas utilissimas. Ávila: 1544.

18. Guerra F. Historia de la Medicina. Barcelona: Capitel; 2007.

19. García Martínez AC, García Martínez MJ. Directorio de enfermeros y artífice de obras de caridad para curar las enfermedades del cuerpo con la práctica de hacer aplicar las medicinas que ordenan los médicos, con el mejor arte y méthodo que ai en ella. Madrid: Consejo General de Enfermería; 2001.

20. Villalba Guitarte J. Epidemiología española o Historia cronológica de las pestes, contagios y epidemias que han acaecido en España desde la venida de los cartagineses hasta el año 1801. 2 vols. Madrid: 1803: 194 y VII.

21. Hernández Morejón A. Historia bibliográfica de la Medicina española. 7 volúmenes Madrid: 1842-1852: XXV.

22. Chinchilla A. Anales históricos de la medicina en general y biográfico-bibliográficos de la española en particular. Valencia: 1841-1847. 
23. Fontana J. La Historia. Análisis del pasado y proyecto social. Barcelona: Crítica; 1982.

24. Casanova J. La Historia Social y los historiadores. Barcelona: Crítica; 2003.

25. León Sanz P. Hacia una historiografía de la enfermedad: Prácticas, profesiones y enfermos. Memoria y Civilización 2008; 11: 243-70.

26. Sigerist H.E., Medicine and human welfare. New Haven: Yale University Press; 1950

27. Ackercknecht EH. History and Geography of the Most Important Diseases. Nueva York: Hafner; 1965.

28. Bourdelais $P$. Histoire de la mèdicine et de la santé: cinquante ans d'expérimentations. Dynamis 1997; 17: 17-36.

29. Bernabeu Mestre J. La actualidad historiográfica de la Historia Social de la Enfermedad. Revista de Demografía Histórica 1989; 7(3): 23-36.

30. Peset JL. De la Historia de la Medicina a la Historia de la Salud. Eidon 2008-2009; 29: 77-83.

31. López Piñero JM. Hacia una Historia Social de la Salud. Aula Hist. Soc. 2000; 6: 510.

32. Siles J, Solana-Ruiz MC, Fernández de Freitas G, Oguisso T. Political Systems and the Perspective on Nursing Education during the Spanish Transition (from the Franco Regime to Democracy). A Contribution based on Nursing History. Aquichan 2010; 10(3):192-203.

33. García- Carpintero Blas E. Reflexión del papel de la enfermería a lo largo de la historia. Enfermería Global (edición digital) 2007; 6 (2). Disponible en: http://revistas.um.es/eglobal/issue/view/70/showToc.

34. Ávila olivares JA. ¿Existió realmente una titulación oficial con el nombre de Ministrante? Cultura de los cuidados 2010; 27: 12-29.

35. Eseverri Chaverri C. Historia de la Enfermería española e hispanoamericana. Barcelona: Salvat; 1984.

36. Santo-Tomás Pérez M. Historia de la Enfermería. En: C. Fernández Ferrín, M. Garrido Abejar y M. Santo-Tomás Pérez (Dirs.). Enfermería Fundamental. Barcelona: Masson; 1994. p. 3-131.

37. Siles González J. Pasado, presente y futuro de la Enfermería en España. Perspectiva Histórica y Epistemológica. Alicante: CECOVA; 1996.

38. Hernández Conesa JM. Historia de la Enfermería. Un análisis histórico de los cuidados de Enfermería. Madrid: McGraw-Hill / Interamericana de España; 1995.

39. Hernández Martín F. (Ed.). Historia de la Enfermería en España (desde la Antigüedad hasta nuestros días). Madrid: Síntesis; 1996.

40. García Martín-Caro C, Martínez Martín ML. Historia de la Enfermería. Evolución histórica del cuidado enfermero. Madrid: Harcourt; 2001.

41. González Canalejo C, Martínez López F. (Eds.) La transformación de la Enfermería. Nuevas miradas para la Historia. Granada: Comares; 2010.

42. Amezcua M. Index y la construcción de una Comunidad de Conocimiento Abierta. Index de Enfermería 2008; 16 (3): 7-10.

43. García Martínez AC, García Martínez M. El modelo Hiades de conocimiento de Historia de la Enfermería. Index de Enfermería 2004; 13 (44): 74-75.

44. Herrera Rodríguez F. Nota sobre la Historia de la Enfermería en España (19772002). Llull. Boletín de Sociedad Española de Historia de la Ciencia 2003; 26: 15773.

45. Sánchez González N, Ortega Martínez C, Elbal Moreno L. Historia de la Enfermería a través de las instituciones de Castilla-La Mancha. Albacete: Colegio de Enfermería; 1996. 
46. Martínez Bruque F, Pérez Luna M. Historia de la Enfermería en Badajoz. Badajoz: Universidad de Extremadura; 2006.

47. Álvarez Nebreda C. Catálogo bibliográfico de publicaciones enfermeras (15411978). Madrid: CODE; 2008.

48. Álvarez Nebreda C. Catálogo de publicaciones periódicas enfermeras 1864-1977. Madrid: CODE; 2010.

49. López Terrada ML. El Hospital como objeto histórico. Los acercamientos a la historia hospitalaria. Revista d' Historia Medieval 1996; 7: 192-204.

50. Gallent Marco M. Historia e historias de los hospitales. Revista d' Historia Medieval 1996; 7: 179-91.

51. Cambil Hernández E. Los Hospitales de Granada. Siglos XVI-XXI. Granada: Universidad de Granada; 2010.

52. Miralles Sangro MT. La Enfermería a través de los sellos. Madrid: DAE; 2008. 